\title{
A Combined Therapy: Anti-Vegf Ocrioplasmin IVT in a Patient with Simultaneous CNV and Vitreomacular Traction
}

\author{
Alessandro Randazzo ${ }^{1}$, Paolo Vinciguerra ${ }^{1}$ and Mary Romano ${ }^{1,2 *}$ \\ ${ }^{1}$ Humanitas Clinical and Research Center, Humanitas University, Italy \\ ${ }^{2}$ Multidisciplinary Department of Medical, Surgical and Dental Sciences, University of Campania Luigi Vanvitelli, Italy \\ Submission: April 27, 2018; Published: September 24, 2018 \\ *Corresponding author: Mary Romano, Department of Ophthalmology, Istituto Clinico Humanitas, Via Alessandro Manzoni 56, 20089 Rozzano, \\ Milan, Italy, Tel: 0039-2-8224-2555; Fax: 0039-2-8224-2554; Email: mary.romano@tin.it
}

\begin{abstract}
Purpose: we report a case of a 76 years old female patient treated with consecutive therapy anti-VEGF-Ocriplasmin for a CNV and vitreomacular traction. She arrives to our observation present a visual loss (3/10) and metamorphopsia in right eye caused by CNV and vitreomacular traction, she has vitreomacular surgery in OS caused by macular hole.

Methods: She was treated with intravireal of aflibercept three injection per month. After 3 injection and after 4month present important visual loss (1/10) OCT showed an important progression of vitreomacular traction, so we decided to treated with Ocrioplasmin IVT.

Conclusions: Consecutive use of Intravitreal drugs to treat different pathologies didn't show side effects or infiammtory reaction caused by pharmacological interaction
\end{abstract}

Keywords: CNV; Vitreomacular traction; Ocrioplasmin; Antivegf

\section{Introduction}

Vitreomacular Traction is an age-related eye condition caused by vitreomacular adhesion. It is observed after the detachment and a part of the vitreous remains firmly attached to the center of the retina. This pulls on the retina and distorts the macula. Edema also occurs and the holes in the macula may form. The central vision is distorted or blurred. The vitreomacular traction can be monitored or treated by surgery only in the most severe cases because of the numerous complications that could generate like retinal detachment and Haemorrhage. Ocriplasmin (Jetrea; Thrombogenics), a recombinant truncated form of human serine protease plasmin with activity against components of the vitreoretinal interface, including fibronectin and laminin, was approved for the treatment of symptomatic VMA [1]. When injected intravitreally, Ocriplasmin induces vitreous liquefaction and separation of vitreoretinal adhesions at the macula and peripapillary retina [2]. Aflibercept is a soluble decoy receptor fusion protein that binds VEGF-A, VEGF-B, and placental growth factor [3.4]. Randomized clinical trials have demonstrated the efficacy of aflibercept in wet age-related macular degeneration [5]. Intravitreal aflibercept is generally well tolerated in patients of different ages with wet age-related macular desease [5].

\section{Case Report}

A 76 year old woman with a history of surgically repaired macular hole in left eye (2010). On May 2011 came for next follow-up one year later surgery. On OCT left eye showed a resolution of macular hole with a residual visus of $2 / 10$, in right eye oct imaging showed a vitreoretinal macular traction $(248$ microns) (Figure 1) asymptomatic with a residual visus of 8/10. Patient decided by herself to don't make any other control.

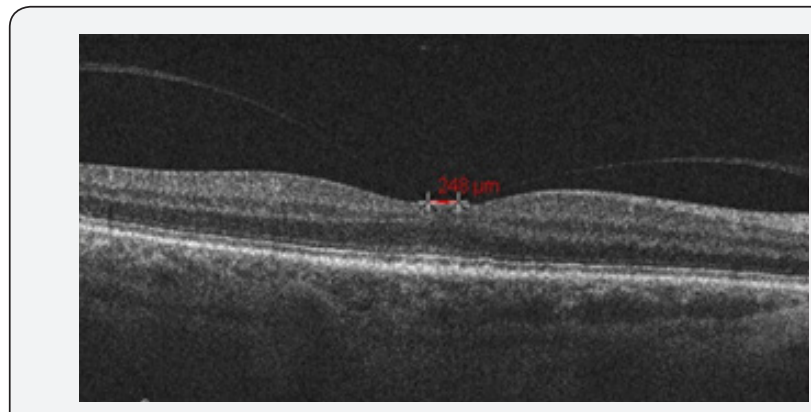

Figure 1: initial VTM traction.

After 5 years she came again to our observation for an important visual loss and metamorphopsia. On examination visual acuity was $3 / 10$, fundus examination showed macular 


\section{JOJ Ophthalmology}

edema and retinal hemmorages, fluorescin angiography (FAG) and optical spectral domain (OCT) showed a classic CNV and a vitreomacular traction (Figure 2). So we decided to start intravitral injection with aflibercept(eleya) [6] one injection for 3 month.

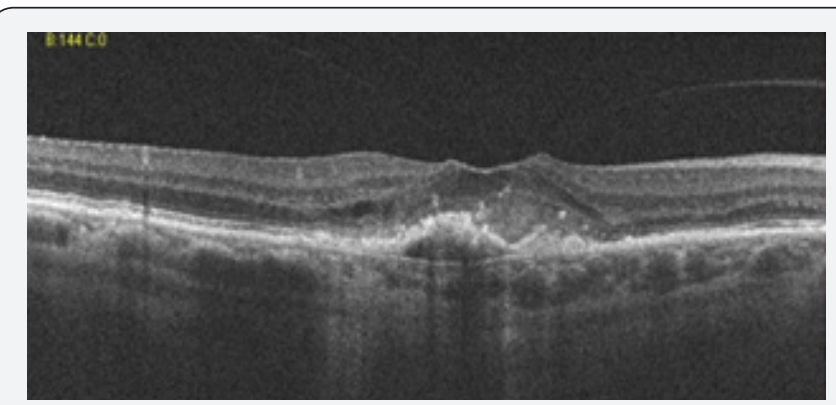

Figure 2: CNV and vitreomacular traction.

Four months later this procedure visus was $1 / 10$ with a resolution of CNV but at follow-up OCT showed a progression of vitreomacular traction (Figure 3). After a thorough discussion of therapeutic option, including surgery or simple observation, the patient elected to undergo injection of ocriplasmin [7].

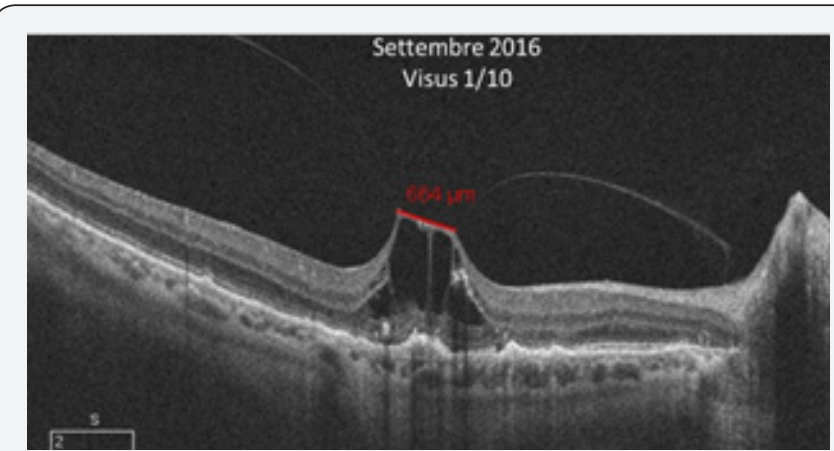

Figure 3: Progression of vitreomacular traction after 4 month from first IVT.

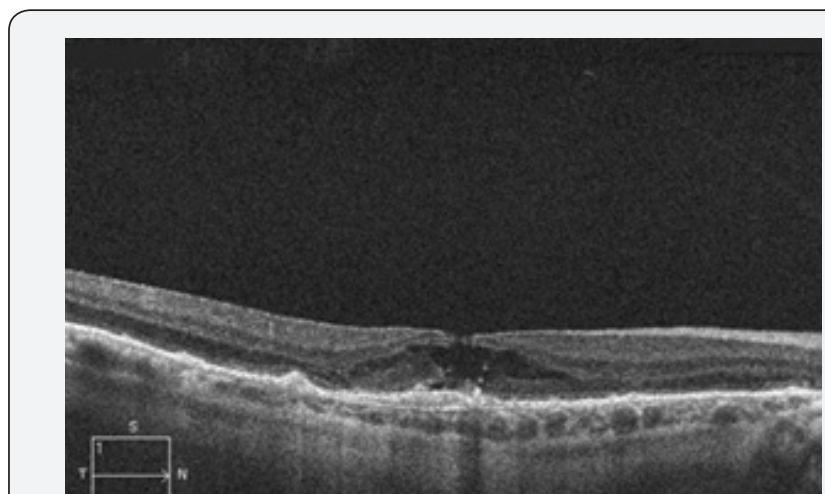

Figure 4: The day after ocrioplasmin IVT.

The patient received one vitreal injection ocrioplasmin at accommodated dose $(0.125 \mathrm{mg}$ in $0.1 \mathrm{ml})$ between two cycle of aflibercept injection. Day after injection OCT showed a completely detachment of vitreal traction associated with lamellar macular hole, no visual effect (Figure 4). After 20 days from ocriplasmin injection OCT demonstrated an atrophic macular hole (Figure 5) with a residual visus of $1 / 10$.

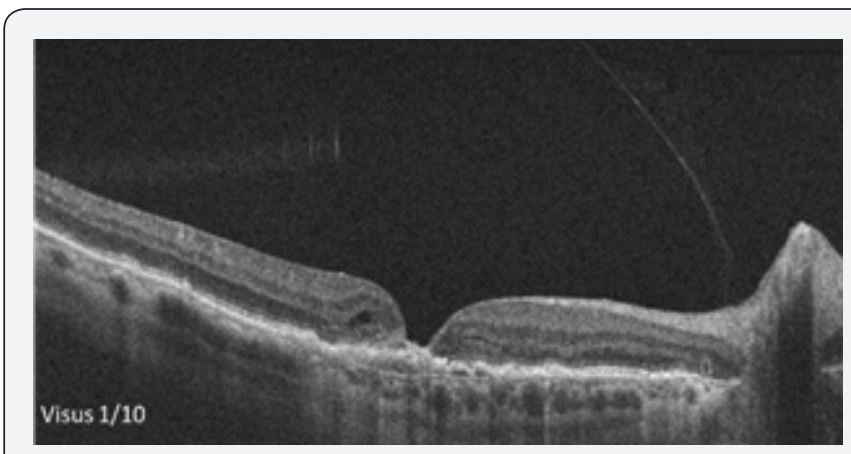

Figure 5: After 1 month from ocrioplasmin IVT.

\section{Discussion}

When deciding on the strategies to be proposed to the patient the therapeutic option was: to keep under review possible spontaneous posterior hyaloid detachment hoping maybe induced by anti VEGF IVT, but this did not happened in previous months and in injections. We think about vitrectomy via pars plana with posterior hyaloid detachment but this could determinated mechanical retinal damage or a phototoxic damage on macula already thinned; so we opted for intravitreal injection of ocriplasmin. Ocrioplasmin IVT seems to be less traumatic on the macula although unknown interaction between anti-VEGF and Ocriplasmin, in fact no formal studies have been conducted on the interaction between the two active drugs. Ocriplasmin, a recombinant truncated form of human serine protease plasmin with activity against components of the vitreoretinal interface, including fibronectin and laminin, was approved for the treatment of symptomatic VMA [8]. When injected intravitreally, Ocriplasmin induces vitreous liquefaction and separation of vitreoretinal adhesions at the macula and peripapillary retina [9]. In pivotal phase 3 clinical trials, a onetime intravitreal injection of Ocriplasmin $(125 \mu \mathrm{g}$ per $100 \mu \mathrm{L})$ was administered in treatment for symptomatic VMA including small (less than or equal to $250 \mu \mathrm{m}$ ) and medium (250 to 400 $\mu \mathrm{m})$ FTMH with persistent VMA [8]. Nobody studies on patient with large macular hole( $>400$ microns), high myopia ( $>8$ D), aphakia, AMD, diabetic retinopathy.

There is no data on concomitant use of ocrioplasmin and Anyi-Vegf in the literature, so we decided to make injection after 30 days each other; so we wait 1 month from third Aflibecept IVT before inject Ocrioplasmin and another month before resuming aflibecept therapy. Unfortunately the precarious and marked thinning of medium and external retinal layers produced as a result of vitreomacular an atrophic Macular hole (Figure 5).

\section{Conclusion}

In summary we present a case of patient start intravitreal treatment for a CNV and in the middle of therapy, after 3 consecutive aflibercept injection, we have to add ocriplasmin injection for treatment of symptomatic vitreomacular traction. We believe it is useful to show how the combined treatment and 
properly spaced two drugs is, at least according to this unique experience, a good therapeutic start without reaction or side effects outcome between the use of two different intravitreal drug.

We hope, on some selected cases, that combined treatment could be a good choice for treatment and safe for patients. The visual acuity of patient remains the same during the time and probably also choosing the surgery the anatomical result was the same. Further studies with association between different intravitreal injection could be made for tasting safety and for the future can be a new therapy approach.

\section{Summary Statement}

We would like to submit to you an original case report that describing the clinical evolution of vitreomacular syndrome in association with CNV and "safety" treatment with two different anti-vegf drugs. We supposed this association is not frequent and it will have an important clinical and therapeutic impact in retinal desease.

\section{References}

1. Haller JA, Stalmans P, Benz MS, Gandorfer A, Pakola SJ, et al. (2015) Efficacy of intravitreal ocriplasmin for treatment of vitreomacular adhesion: Subgroup analyses from two randomized trials. Ophthalmology 122(1): 117-122.
2. Kim BT, Schwartz SG, Smiddy WE, Doshi RR, Kovach JL, et al. (2013) Initial outcomes following intravitreal ocriplasmin for treatment of symptomatic vitreomacular adhesion. Ophthalmic Surg Lasers Imaging Retina 44(4): 334-343.

3. Papadopoulos N, Martin J, Ruan Q Rafique A, Rosconi MP, et al. (2012) Binding and neutralization of vascular endothelial growth factor (VEGF) and related ligands by VEGF Trap, ranibizumab and bevacizumab. Angiogenesis 15(2): 171-185.

4. Deissler HL, Lang GK, Lang GE (2014) Capacity of aflibercept to counteract VEGF-stimulated abnormal behavior of retinal microvascular endothelial cells. Exp Eye Res 122: 20-31.

5. Rosenfeld PJ, Brown DM, Heier JS, Boyer DS, Kaiser PK, et al. (2006) Ranibizumab for neovascular age-related macular degeneration. N Engl J Med 355(14): 1419-1431.

6. Duval MV, Rougier MB, Delyfer MN, Combillet F, Korobelnik JF (2017) [Real Life Visual And Anatomic Outcomes Of Aflibercept Treatment For Treatment-Naive Patients With Exudative Age-Related Macular Degeneration]. J Fr Ophtalmol 40(4): 270-278.

7. Adamson P, Wilde T, Dobrzynski E, Sychterz C, Polsky R, et al. (2016) Single ocular injection of a sustained-release anti-VEGF delivers 6 months pharmacokinetics and efficacy in a primate laser $\mathrm{CNV}$ model. J Control Release 244(Pt A): 1-13.

8. Stalmans P, Benz MS, Gandorfer A, Kampik A, Girach A, et al. (2012) Enzymatic vitreolysis with ocriplasmin for vitreomacular traction and macular holes. N Engl J Med 367(7): 606-615.

9. Kuppermann BD (2012) Ocriplasmin for pharmacologic vitreolysis. Retina 32 Suppl 2: S225-S228. 